\title{
Water Nanocondensation on Polymer Single Crystal-Decorated Buckypaper
}

\author{
Eric. D. Laird, ${ }^{\dagger} \mathrm{Hao}$ Qi, and Christopher Y. $\mathrm{Li}^{*}$ \\ Department of Materials Science and Engineering, Drexel University, \\ Philadelphia, Pennsylvania, 19104, U.S.A. \\ ${ }^{\dagger}$ Present Address: RDRL-WMM-G, 4600 Deer Creek Loop, Aberdeen Proving \\ Ground, Maryland 21005-5069, U.S.A.
}

*corresponding author, E-mail: chrisli@drexel.edu

Keywords: Polymer wetting, carbon nanotubes, nanocondensation, polymer

crystallization, nano hybrid shish kebab

\begin{abstract}
Condensation on the surfaces of single-walled carbon nanotube (SWCNT) buckypaper and polymer single crystal-decorated buckypaper was monitored in situ using an environmental SEM (ESEM). Water vapor was pumped into the chamber, and backside cooling was used to control sample surface temperature. In this way, condensation could be imposed on the sample. It was found that, due to the different wetting states, condensation contact angles of all the samples are significantly lower than the static contact angles measured by the sessile drop method. The polyethylene (PE) single crystal-decorated buckypaper also strongly resist water vapor condensation, thereby precluding flooding below the fog point. Our results show that the heterogeneous surface structure plays a key role in determining water condensation.
\end{abstract}




\section{Introduction}

Carbon nanotube (CNT)-based membranes and coatings have shown considerable merit for use in both the control and sensing of humidity.[1-6] CNT-based surfaces have been used to detect analytes from the gas phase[7-9] and functionalized to control wetting behavior.[10, 11] Water vapor rejection has also been demonstrated: aligned CNTs have been shown to selectively reject water vapor from a gas mixture.[6] To explain this behavior, a three-stage condensation, agglomeration, rejection mechanism whereby water droplets nucleate and are collected on impurities until they can roll off of the barrier into a collection vessel, has been suggested. In the case of semicrystalline polymers, water wetting behavior is sensitive to crystalline structure and morphology.[12, 13] We have previously shown that by decorating CNTs with polymer single crystals,[14-16] a novel hybrid material could be produced which was termed "nanohybrid shish kebab" (NHSK), where CNTs served as the shish and polymer single crystals formed kebabs on the CNTs.[15] Simple vacuum filtration of NHSK dispersions allowed the formation of freestanding NHSK paper that mimics buckypaper, with controlled pore sizes. The NHSK paper is electronically conductive with controllable water wetting because of the hierarchical roughness and well-tuned local chemistry.[17] Further modifying these materials with PTFE broadened the range of wetting behaviors that could be accessed, suggesting additional applications.[18, 19]

To better understand the wetting behavior of water on NHSK paper, it is desired to investigate the condensation process in situ. Previous work showed that wetting under ESEM closely simulates the conditions in the vicinity of a cathode catalyst layer.[20] Yu et al. and Zhang et al. used this technique to explore the conditions at the catalyst layer.[21, 22] 
Addressing the general issue of microcondensation on rough surfaces, Jung and Bhushan used ESEM to study droplet formation on wafers patterned with pillars of different geometry and pitch coated with a monolayer of 1,1,-2,2,-tetrahyrdoperfluorodecyltrichlorosilane, and showed how roughness impacts the formation of condensation drops.[23] ESEM has also been used to study water condensation on lotus leaves and to study spatial control of heterogeneous nucleation of water.[24-26] In this paper, the behavior of water molecules condensed from the vapor phase on SWCNT buckypaper and NHSK paper was explored. We show that incorporating polymer single crystals onto SWCNT sidewalls is an effective means to tune wetting behavior and detailed analysis of ESEM wetting behaviors revealed how water condensation was affected.

\section{Experimental}

\subsection{Preparing NHSK paper}

Figure 1 shows the experimental process. High-density PE (MFI 12 g/10 min), 1,2dichlorobenzene (DCB, 99\% manufacturer's spec.), isopropanol, and rinse solvents were purchased from Aldrich and used as received. SWCNT, "purified" grade, were purchased from Unidym. In a typical experiment, $24 \mathrm{mg}$ SWCNT would be placed in a $250 \mathrm{~mL}$ round-bottom flask with $120 \mathrm{~g} \mathrm{DCB}$, in the expectation that the final crystallization solution would contain approximately $50 \%$ of the original CNT content and $85 \%$ of the original DCB (0.01 wt.\% SWCNT). 2 vol.\% isopropanol was added to prevent sonopolymer functionalization of the CNTs. Separately, a desired quantity of PE was added to a $100 \mathrm{~mL}$ round bottom flask with an appropriate amount of DCB, purged with $\mathrm{N}_{2}$, and heated to dissolution under a $\mathrm{N}_{2}$ blanket. The CNT solution was bath sonicated for $90 \mathrm{~min}$, tip sonicated for an additional $30 \mathrm{~min}$, and then divided up into centrifugation vials and centrifuged for $20 \mathrm{~min}$ at 10,000 rpm. The supernatant 
was collected and equilibrated to the $\mathrm{PE}$ dissolution temperature $\left(130^{\circ} \mathrm{C}\right)$ for $8-10 \mathrm{~min}$. The $\mathrm{PE}$ solution was then cast into the CNT dispersion and the combined solution was mixed for 3 min before quenching to the crystallization temperature, $88.5^{\circ} \mathrm{C}$, and would remain at this temperature for $120 \mathrm{~min}$. Isothermal filtration was performed $3 \mathrm{x}$ after the initial rinse. The filtered solution was then quenched to room temperature. Samples were filtered over $0.2 \mu \mathrm{m}$ PTFE membranes from Savillex. For pure buckypaper, this filtration step had to be done immediately after centrifugation to mitigate aggregation of the CNTs.
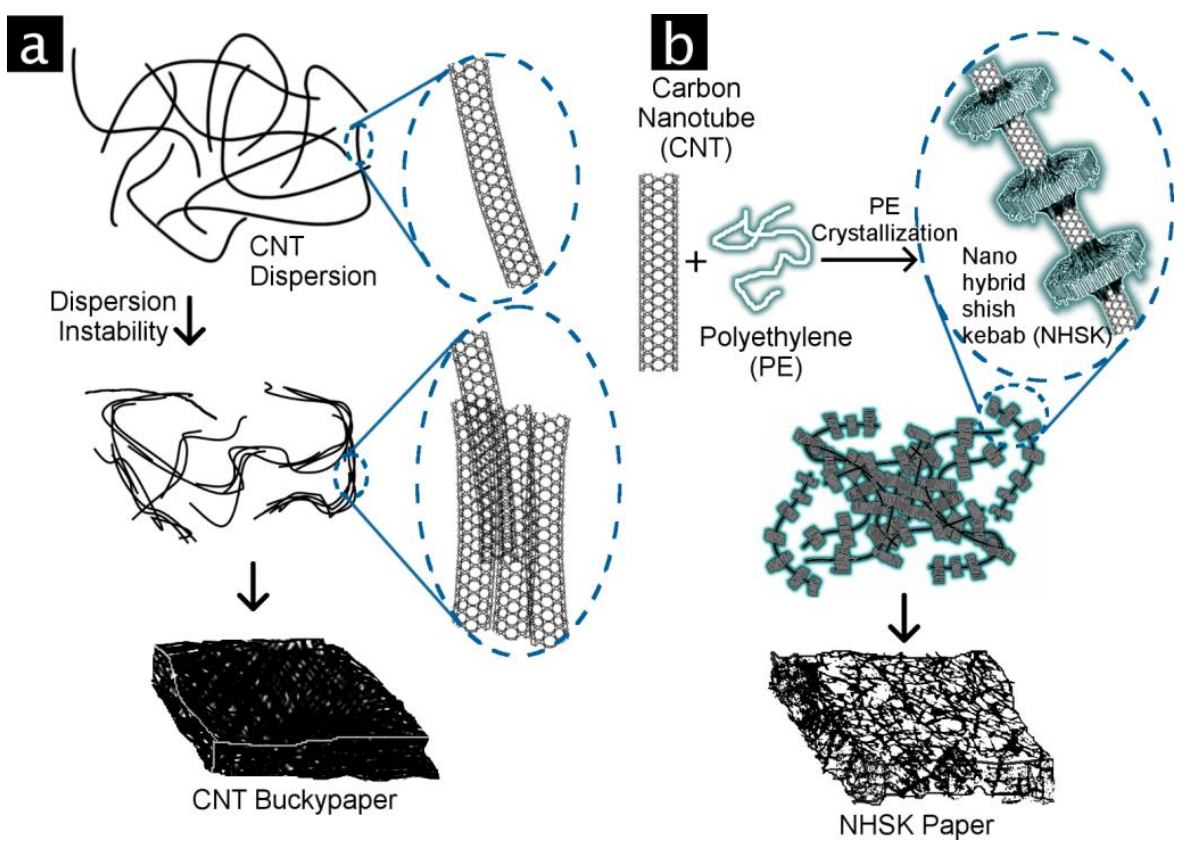

Figure 1. Deposition of SWCNT buckypaper and NHSK paper. a) Dispersed CNTs flocculate in solution during vacuum filtration. A nonwoven mat of SWCNT bundles is thus produced. b) Solution crystallization of PE forms a physical functionalization to prevent reaggregation of SWCNTs. The initial ratio of PE to SWCNT in solution dictates the kebab size. This hybrid structure is termed "nano hybrid shish kebab" (NHSK). Vacuum filtration of NHSK produces a porous nonwoven mat with regular features on the 10-100 nm length scale, termed "NHSK paper." 


\subsection{Characterization}

Surface characterization of the SWCNT buckypaper and NHSK paper was done on a Zeiss

Supra 50VP operating at $1 \mathrm{keV}$ in secondary electron detection mode. In situ characterization of buckypaper and NHSK paper surfaces was performed in an FEI XL30 Environmental SEM in wet mode using a cooling stage from Peltier. Samples were stuck to the stage by first wiping on a small amount of vacuum grease and then gently unfurling the sample over the greased surface. The sample was cooled to $1^{\circ} \mathrm{C}$ and recordings were made during a step-ramp pressure increase. Starting at a vapor pressure of 3.0 torr ( $62 \%$ relative humidity), the pressure was ramped 0.1 torr/min until condensation would appear on the surface and remain on the surface without evaporating. Sessile drop contact angles (CAs) were recorded on a Sony XC-HR90 camera with a C-mount lens. A minimum of 5 droplets (using $10 \mu \mathrm{L}$ droplets of distilled water from Aqua Solutions, Inc.) were individually measured to analyze sessile drop CAs.

\section{Results and Discussion}

\subsection{Surface Properties of SWCNT Buckypaper and NHSK papers}

Figure 2 shows the appearances of SWCNT buckypaper and polymer single crystaldecorated SWCNT paper surfaces. It can be seen that the SWCNT buckypaper (Figure 2a) forms a dense mat with openings dotting the surface: this is due to SWCNT bundling and SWCNTs connecting separate bundles as tie chains, forming a web-like network. Decorating CNTs with PE single crystals to form NHSK structures provides functionalization, allowing tube-tube separation.[16, 27-36] Vacuum filtration of the NHSK suspension led to the formation of porous buckypaper analogs called "NHSK paper." Samples designated as NHSK- $n$, where $n$ is the percentage of CNTs in the sample, are shown in Figures 2c,e,f. With increasing PE content from 
Figures $2 \mathrm{c}$ to $2 \mathrm{e}$, the surface roughness increases and the static contact angle (sCA) increases from $117.8^{\circ}$ to $138.0^{\circ}$. Further increasing the PE content by increasing PE lamellar size leads to the mechanical instability of the PE crystallites, which in turn, causes the surface features to collapse. This is reflected in the shapes of sessile drops placed on the surfaces of these films and the sCA decreases to $117.1^{\circ}$. SWCNT buckypaper is slightly hydrophilic. The sCA of buckypaper was found to be $83.6 \pm 2.7^{\circ}$. NHSK papers are statically hydrophobic and dynamically non-sliding. This has been attributed to the nanoscale Cassie and micro-scale Wenzel wetting behavior, whereupon the shish kebab "posts" on NHSK paper effectively exclude water from the interkebab galleries (Figure 2d). sCAs for each surface are given in Table 1.

Table 1. sCAs (uncertainty taken from standard deviation of measurements of at least 5 droplets), advancing and receding cCAs (description in text; each distribution calculated from $\sim 50$ droplets), and pressure required for the onset of stable condensation $\left(P_{\mathrm{co}}\right)$ along with corresponding relative humidity extrapolated from isobar data at the sample cooling temperature.

\begin{tabular}{|c|c|c|c|c|c|}
\hline & sCA $\left(^{\circ}\right)$ & $\begin{array}{c}\text { Advancing cCA } \\
\left({ }^{\circ}\right)\end{array}$ & $\begin{array}{c}\text { Receding cCA } \\
\left({ }^{\circ}\right)\end{array}$ & $\begin{array}{c}P_{\text {co }} \\
\text { (torr) }\end{array}$ & $\begin{array}{c}\text { Relative } \\
\text { humidity } \\
\text { at } P_{\text {co }}(\%)\end{array}$ \\
\hline $\begin{array}{c}\text { SWCNT } \\
\text { buckypaper }\end{array}$ & $83.6 \pm 2.7$ & $40.6+7.7 /-9.2$ & $31.2+9.3 /-13.0$ & 4.4 & 89.5 \\
\hline NHSK-54 paper & $\begin{array}{c}114.1 \pm \\
6.0\end{array}$ & $70.6+12.0 /-13.0$ & $24.5+9.7 /-17.5$ & 4.8 & 97.5 \\
\hline NHSK-38 paper & $\begin{array}{c}137.9 \pm \\
4.1\end{array}$ & $88.0+6.9 /-7.0$ & $\begin{array}{c}53.4+14.1 /- \\
17.5\end{array}$ & 5.3 & 105.5 \\
\hline NHSK-14 paper & $\begin{array}{c}125.7 \pm \\
8.7\end{array}$ & $84.1+15.3 /-15.8$ & $\begin{array}{c}50.9+17.0 /- \\
23.1\end{array}$ & 6.1 & 123.4 \\
\hline
\end{tabular}



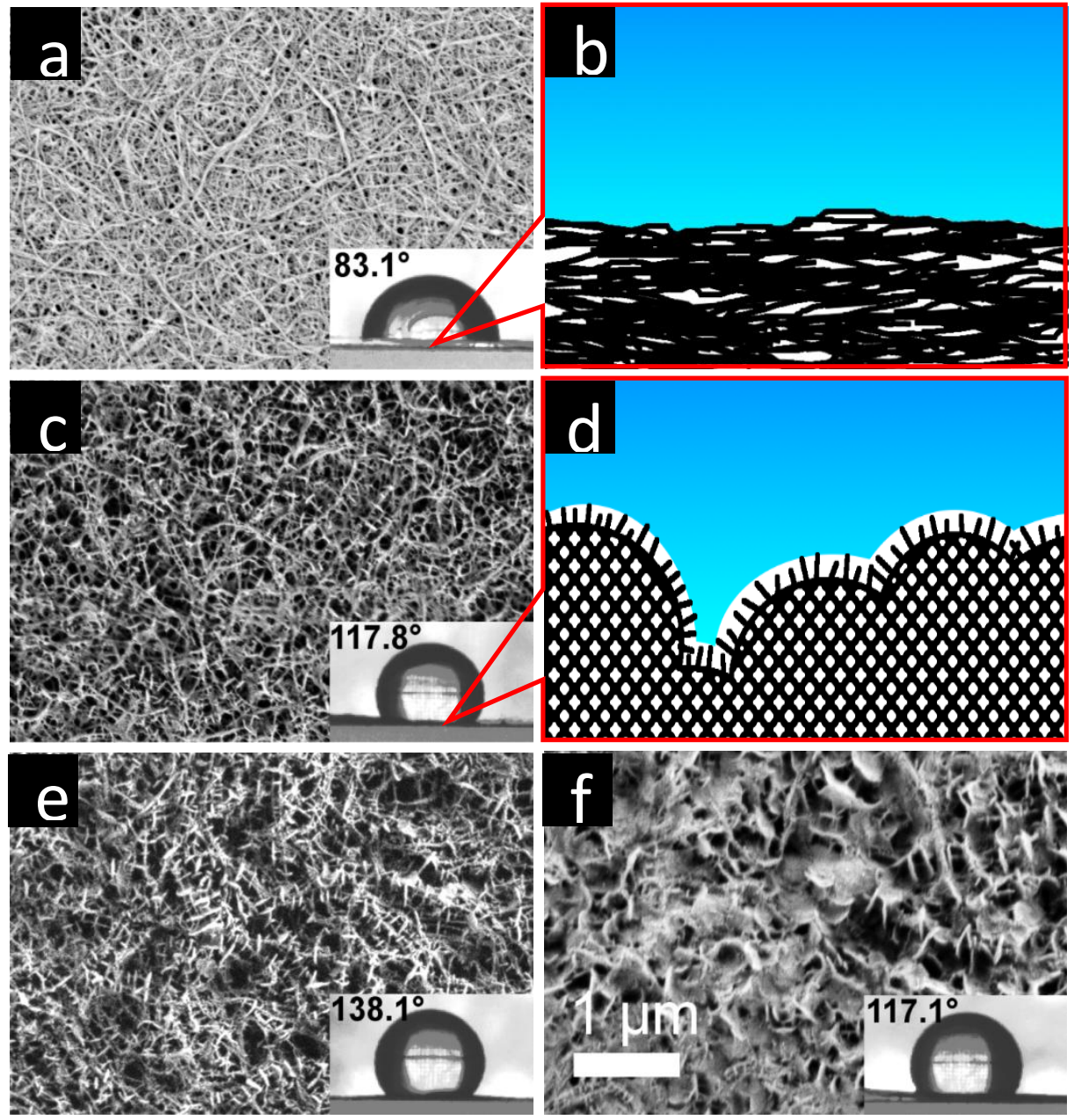

Figure 2. Surface characteristics of SWCNT buckypaper and NHSK paper. a,c,e,f) 10,000x magnification micrographs of top surfaces of SWCNT buckypaper, and NHSK paper with 54 wt.\%, 38 wt.\%, and 14 wt.\% CNT, respectively. Inset in a,c,e,f: shapes of $10 \mu \mathrm{L}$ sessile droplets on the surfaces of each paper. b) Schematic representation of water/buckypaper interface. d) Schematic representation of the water/NHSK paper interface. 


\subsection{Condensation on SWCNT and NHSK papers}

While much information can be derived from sessile droplets and other types of macroscale experiments, wetting behavior can be better understood by studying controlled water nanocondensation using ESEM. For example, ESEM wet mode allows femtoliter quantities to be manipulated and studied easily. The droplets can also be monitored in situ. In this study, an ESEM was thus used to explore the applicability of NHSK paper for condensation control. Using wet mode operation, condensation droplets in the femto- to picoliter range could be observed. An example of the progression of a typical experiment is shown in Figure 3. (Experimental conditions at each frame of Figure 3 are indicated in Table S1 in the Supporting Information.) Figure 3a shows a SWCNT buckypaper surface in the chamber prior to the introduction of moisture. After every ten seconds of the experiment the environment pressure was stepped up by 0.1 torr to lower the gas solubility of water in the chamber. After approximately $155 \mathrm{~s}$, the first traces of condensation could be observed on the surface of the buckypaper. As the experiment continued, transient condensation droplets dotted the surface and then were re-evaporated as the environmental conditions stabilized (see, e.g., Figures 3c-d and 3e-f). Eventually, the environmental conditions in the chamber reached a point where condensation droplets continually and irreversibly swelled (Figure 3j), representing the point where the sample became saturated. After a 1 minute period of holding the ESEM at the saturation condition (Figure $3 \mathrm{j}-\mathrm{u}$ ), the operating pressure was reduced to allow the condensation droplets to recede (Figure $3 \mathrm{v}-\mathrm{x}$ ) thereby allowing receding cCAs to be recorded. 

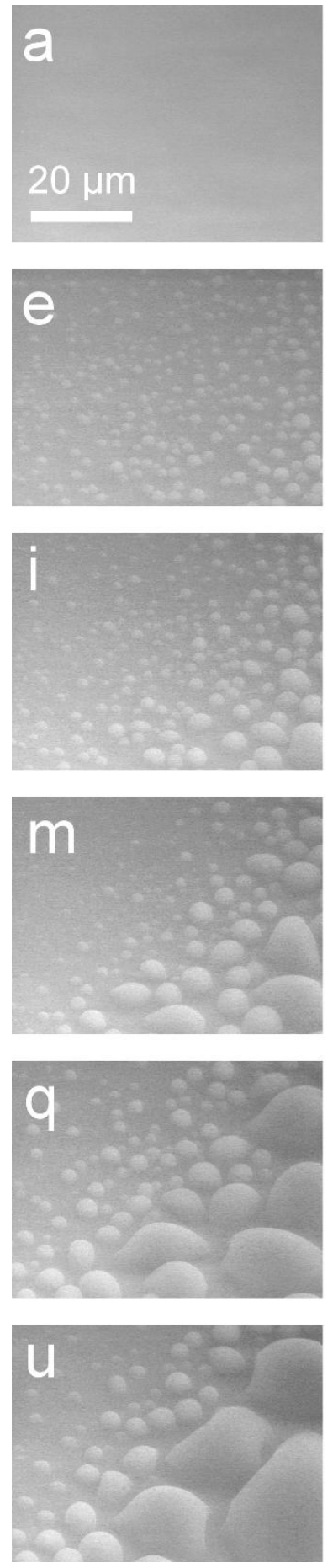
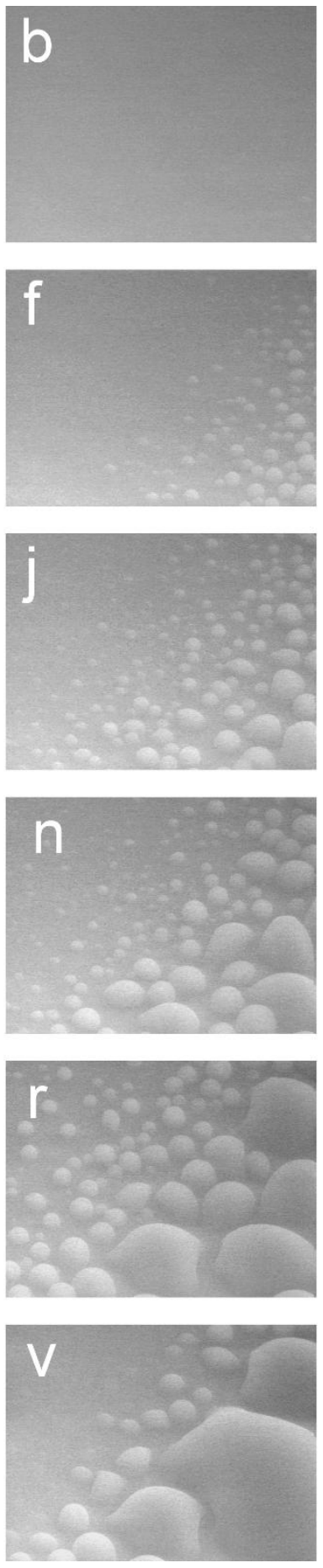
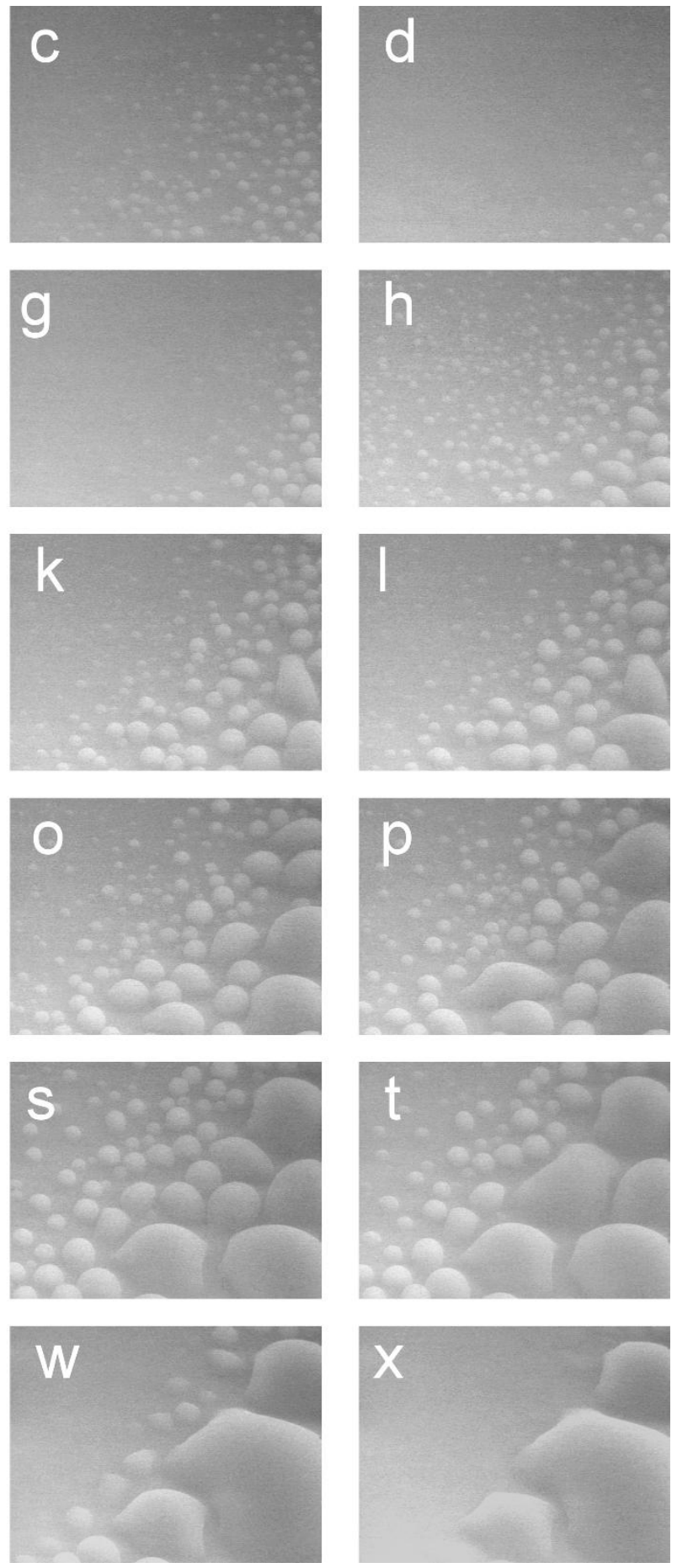

Figure 3. Appearances of droplets on SWCNT buckypaper in a typical ESEM experiment. Video recordings of the experiment are available in Supporting Information. Tabulated environmental operating conditions are provided in Table 2 . The experimental humidity profile is also available in Supporting Information, Figure S3. 
Table 2. Time and ESEM vapor pressure for stillframe images shown in Figure 3.

\begin{tabular}{|l|l|l|l|}
\hline $\begin{array}{l}\mathbf{a} \\
\text { Time }-0: 00 \\
\text { Pressure }-2.9 \text { torr }\end{array}$ & $\begin{array}{l}\mathbf{b} \\
\text { Time }-2: 37 \\
\text { Pressure }-4.4 \text { torr }\end{array}$ & $\begin{array}{l}\mathbf{c} \\
\text { Time }-2: 41 \\
\text { Pressure }-4.5 \text { torr }\end{array}$ & $\begin{array}{l}\mathbf{d} \\
\text { Time }-2: 46 \\
\text { Pressure }-4.5 \text { torr }\end{array}$ \\
\hline $\begin{array}{l}\mathbf{e} \\
\text { Time }-2: 51 \\
\text { Pressure }-4.6 \text { torr }\end{array}$ & $\begin{array}{l}\mathbf{f} \\
\text { Time }-2: 56 \\
\text { Pressure }-4.6 \text { torr }\end{array}$ & $\begin{array}{l}\mathbf{g} \\
\text { Time }-3: 00 \\
\text { Pressure }-4.6 \text { torr }\end{array}$ & $\begin{array}{l}\mathbf{h} \\
\text { Time }-3: 02 \\
\text { Pressure }-4.7 \text { torr }\end{array}$ \\
\hline $\begin{array}{l}\mathbf{i} \\
\text { Time }-3: 05 \\
\text { Pressure }-4.7 \text { torr }\end{array}$ & $\begin{array}{l}\mathbf{j} \\
\text { Time }-3: 08 \\
\text { Pressure }-4.7 \text { torr }\end{array}$ & $\begin{array}{l}\mathbf{k} \\
\text { Time }-3: 13 \\
\text { Pressure }-4.7 \text { torr }\end{array}$ & $\begin{array}{l}\text { Iime }-3: 17 \\
\text { Pressure }-4.7 \text { torr }\end{array}$ \\
\hline $\begin{array}{l}\text { mime }-3: 20 \\
\text { Pressure }-4.7 \text { torr }\end{array}$ & $\begin{array}{l}\mathbf{n} \\
\text { Time }-3: 24 \\
\text { Pressure }-4.7 \text { torr }\end{array}$ & $\begin{array}{l}\mathbf{0} \\
\text { Time }-3: 27 \\
\text { Pressure }-4.7 \text { torr }\end{array}$ & $\begin{array}{l}\mathbf{p} \\
\text { Time }-3: 31 \\
\text { Pressure }-4.7 \text { torr }\end{array}$ \\
\hline $\begin{array}{l}\mathbf{q} \\
\text { Time }-3: 35 \\
\text { Pressure }-4.7 \text { torr }\end{array}$ & $\begin{array}{l}\text { Time }-3: 40 \\
\text { Pressure }-4.7 \text { torr }\end{array}$ & $\begin{array}{l}\mathbf{s} \\
\text { Time }-3: 49 \\
\text { Pressure }-4.7 \text { torr }\end{array}$ & $\begin{array}{l}\text { Time }-3: 59 \\
\text { Pressure }-4.8 \text { torr }\end{array}$ \\
\hline $\begin{array}{l}\text { Time }-4: 07 \\
\text { Pressure }-4.8 \text { torr }\end{array}$ & $\begin{array}{l}\text { Time }-4: 14 \\
\text { Pressure }-4.6 \text { torr }\end{array}$ & $\begin{array}{l}\mathbf{w} \\
\text { Time }-4: 24 \\
\text { Pressure }-4.4 \text { torr }\end{array}$ & $\begin{array}{l}\mathbf{x} \\
\text { Time }-4: 43 \\
\text { Pressure }-4.4 \text { torr }\end{array}$ \\
\hline
\end{tabular}

To explore the applicability of NHSK paper for condensation control, the behavior of condensation at the surfaces of these films was also studied using an ESEM. Examples of a series of still images recorded from in situ ESEM condensation experiments on SWCNT buckypaper and NHSK-54 paper are shown in Figure 4, along with rose histograms of condensation $\mathrm{CA}(\mathrm{cCA})$ data. For the measurement of cCAs, sample surfaces were angled towards the gaseous detection device (GDD) such that forward edges of the condensation droplets' contact circles could be observed, from which the cCA could be calculated (derivation available in the Supporting Material, Figures S1, S2). If the droplet oriented at a tilt angle $\phi$ can be assumed to form a spherical cap with radius $R$ and contact circle radius $a$, it will appear to the viewer to have a total "observable altitude" $B$, defined as the distance from the top of the 
observed arc of the spherical cap to the point on the contact circle closest to the GDD. $a$ can be calculated from the quadratic equation as

$$
a^{2}\left(\sin ^{2} \phi+\cos ^{2} \phi\right)+a(2 R \cos \phi-2 B \cos \phi)+\left(B^{2}+R^{2}-R^{2} \sin ^{2} \phi-2 B R\right)=0 .
$$

Calculation of $a$ allows for the determination of the cCA.[37] Noting that if $B>R(1+\cos \phi)$, $\theta>90 ;$ the cCA can be written in closed form as

$$
\theta= \begin{cases}2 \tan ^{-1} \frac{R}{a}\left(1+\sqrt{1-\frac{a^{2}}{R^{2}}}\right) & \text { if } B>R(1+\cos \phi), \\ 2 \tan ^{-1} \frac{R}{a}\left(1-\sqrt{1-\frac{a^{2}}{R^{2}}}\right) & \text { if } B \leq R(1+\cos \phi) .\end{cases}
$$

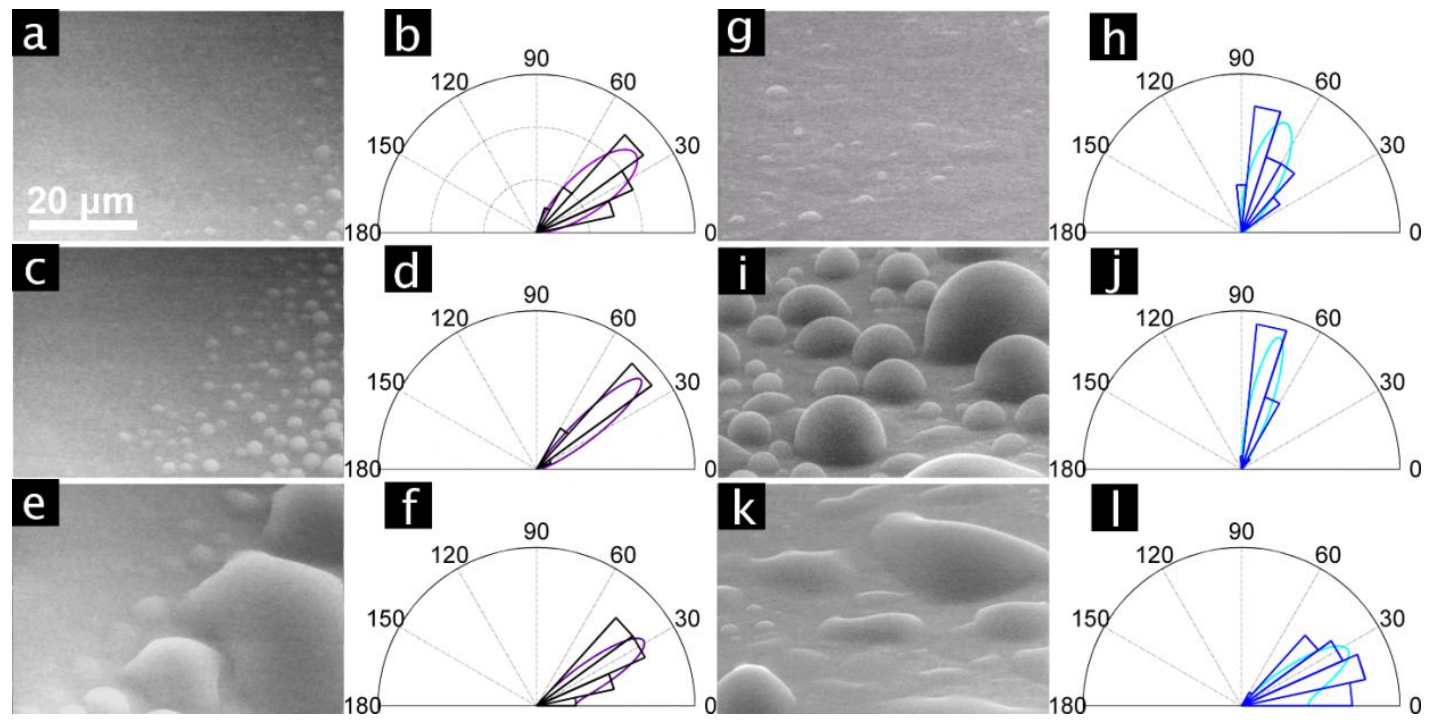

Figure 4. Development of condensation droplets on (a-f) SWCNT buckypaper and (g-1) NHSK-54 paper. All images and histograms are taken past the onset of stable condensation. SWCNT buckypaper shown at a) 4.5 torr, $92 \%$ relative humidity; c) 4.6 torr, $94 \%$ relative humidity, and e) 4.4 torr, $90 \%$ relative humidity. b, d, f) Corresponding incipient (b), advancing (d), and receding (f) histograms and best-fit probability loops for SWCNT buckypaper. g,i,k) 
NHSK-54 paper shown at g) 4.8 torr, $98 \%$ relative humidity; i) 4.9 torr, $100 \%$ relative humidity; and k) 4.5 torr, $90 \%$ relative humidity. h,j,l) Corresponding incipient (h), advancing (j), and receding (l) cCA histograms and best-fit probability loops for NHSK-54 paper.

Table 1 shows summarized data from sCA and cCA experiments, as well as a summary of the conditions necessary for the onset of condensation. Stable condensation was recorded as the lowest pressure where droplets were sustained through the quasi-static conditions in the chamber until the next incremental pressure increase. After the onset of stable condensation, a maximum average CA was eventually reached which was considered as the advancing cCA. Above this point, flooding was virtually unavoidable, but by lowering the ambient pressure evaporation could be used to minimize droplet coalescence. Receding cCAs were calculated as the minimum average droplet $\mathrm{cCA}$ as the pressure was lowered below the point of supersaturation. In analyzing the data to determine standard deviations, it was assumed that the droplets' angle cosines would follow Gaussian statistics. Re-converting from the linearized cosine form causes the distributions to appear skew normal.

A few particular points related to the wetting behavior shown in Figure 4 are worthy of note. Most importantly, the cCAs were, in general, much lower than those measured by the sCA technique. Chemical and morphological inhomogeneities of NHSK films, combined with differences between the experimental details of these two techniques, serve to artificially increase the sCA while deflating the cCA. NHSK papers are statically hydrophobic but dynamically non-sliding. The hierarchical roughness is due to inter- and intra-NHSKs. The quasi-periodic arrangement of the kebab crystals with a $50 \mathrm{~nm}$ kebab spacing contributes to the nanoscale roughness; 2D random packing of these NHSKs leads to an inter-NHSK roughness 
that is $\sim$ a few hundred nanometers. In a sessile droplet measurement, the edges of a water droplet are pinned as a water droplet settles onto the surface, trapping air pocket underneath and making the measured sCA higher than they would be at equilibrium (Figure 1e, inset). On the other hand, three effects are responsible for lowering the cCA: 1) Water adsorbs weakly to CNT sidewalls and to the creases in CNT bundles.[38, 39] This suggests that as pressure within the SEM increases, a diffuse layer of water adsorbs on the CNT/NHSK paper surface even before actual water beads begin to form.[40] The "air pockets" formed in sCA are therefore absent in the cCA measurement. 2) The droplets formed in an ESEM are not at strict equilibrium. Ostwald ripening allows larger diameter drops to swell at the expense of smaller ones. Because the distribution includes an unweighted average of individual droplet cCAs, the final result can be lowered by the relatively large number of small droplets that are siphoned by the ripening process. 3) Droplet coalescence is also responsible for lowering the average cCA. In this case, because of the naturally low receding CA of NHSK paper,[17] coalescing droplets will remain pinned at the outermost extremes of the contact circle boundaries. Thus, while the contact line might try to reorient to approach an equilibrium value, the pinning effect will dominate the behavior of droplets organizing at the surface.[41][42]

Advancing cCAs follow a trend that mimics that of the sCA in that increasing the PE loading first increases, then decreases, following the nanoscale surface roughness.[17] Still, sCAs were found to be an average of $45^{\circ}$ higher than cCAs. Nanocondensation droplet distributions were found to be exceptionally broad relative to the behavior of sessile drops. The reason for this is expected to be due to a combination of Ostwald ripening and droplet coalescence. A schematic representation of the expected mechanism of incipient droplet formation is shown in Figure 5. The surface energy of the polymer single crystal fold surface is relatively low: for PE it is 
expected to be $\sim 104^{\circ}$.[43] Therefore, a cluster of water molecules is effectively confined to an intrakebab gallery. An incipient water droplet can only block a gas feed if it can form water wicks with droplets initiated at adjacent NHSK, so droplet confinement can effectively mitigate sample flooding if a liquid phase pressure gradient is imposed. Conversely, with SWCNT buckypaper, no polymer single crystals are present and so the relatively small pores that exist are quickly flooded. Condensation can occur in these films even below vapor phase saturation given a small change in temperature.
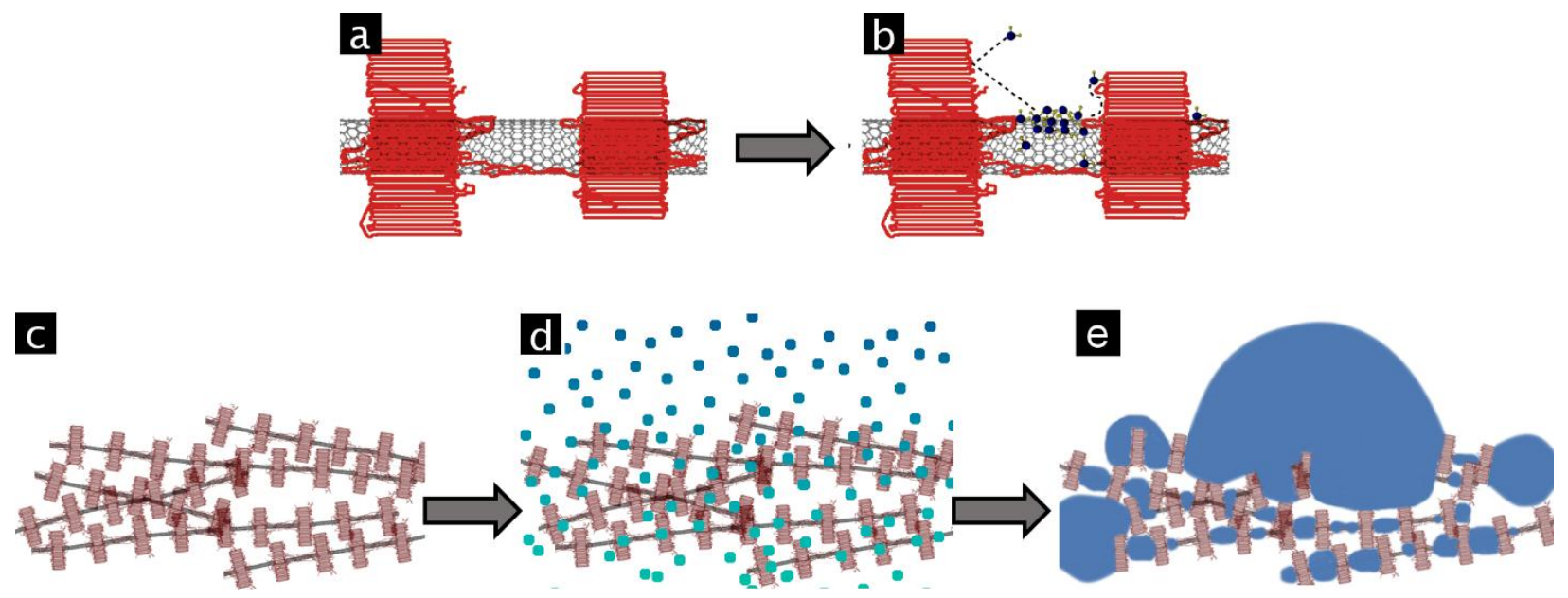

Figure 5. Proposed mechanism of condensation formation at a NHSK surface. a) Intrakebab gallery (drawing not to scale). b) Water molecules collect at the exposed CNT surface. c) NHSK paper prior to saturation with water vapor. d) Water vapor infiltrates NHSK paper owing to NHSK's high permeability. e) NHSK paper surface confines incipient condensation droplets in intrakebab galleries, with few CNTs in close enough proximity to form intersections that would allow water wicks to develop.

From the preceding discussion it also becomes clear why the relative pressure required for condensation increases with increasing polymer content. As the aspect ratio of the intrakebab asperity increases, it becomes increasingly difficult to collect water molecules at the CNT 
surface. In the limit of increased PE loading to the extent that the single crystals collapse, vapor phase water molecules must explore deep into the interior of the NHSK paper to find a CNT on which to agglomerate. This implies that for unmodified NHSK papers, a tradeoff exists between conductivity and flooding resistance.

\section{Conclusions}

We have fabricated a unique type of NHSK paper using vacuum filtration. The polymer single crystals on the NHSK paper surface control the film roughness, and the correspoinding water condensation behavior. We show that incorporating polymer single crystals onto SWCNT sidewalls is an effective means to tune wetting behavior. In situ nanocondensation study using ESEM and the detailed analysis of wetting behaviors revealed how water condensation was affected. The cCAs of said microdroplets followed the general trends of macroscopic droplets, but were significantly lower in all cases owing to subsurface origination. Notably, the effect of incorporating polymer single crystals onto CNTs was found to be highly effective in preventing water condensation.

\section{ACKNOWLEDGMENTS}

This work was funded by NSF grants DMR-1308958 and the NSF-IGERT DGE-0221664. The authors wish to thank Dr. Ed Basgall of the Drexel University Centralized Research Facility for training and assistance in the use of the ESEM. 


\section{REFERENCES}

1. Modi A, Koratkar N, Lass E, Wei B, and Ajayan PM. Nature 2003;424(6945):171-174.

2. Zhang Y, Yu K, Xu R, Jiang D, Luo L, and Zhu Z. Sensors and Actuators A: Physical 2005;120(1):142-146.

3. Jung D, Han M, and Lee GS. Materials Letters 2014;122(0):281-284.

4. Trojanowicz M. TrAC Tren. Anal. Chem. 2006;25(5):480-489.

5. Kauffman DR and Star A. Angew. Chem. Intern. Ed. 2008;47(35):6550-6570.

6. Yoon D, Lee C, Yun J, Jeon W, Cha BJ, and Baik S. ACS Nano 2012;6(7):5980-5987.

7. Sinha N, Ma J, and Yeow JTW. J. Nanosci. Nanotechn. 2006;6(3):573-590.

8. Varghese OK, Kichambre PD, Gong D, Ong KG, Dickey EC, and Grimes CA. Sens. Actuat. B: Chemical 2001;81(1):32-41.

9. Snow ES, Perkins FK, Houser EJ, Badescu SC, and Reinecke TL. Science 2005;307(5717):1942-1945.

10. Gupta M, Kapur V, Pinkerton NM, and Gleason KK. Chem. Mater. 2008;20(4):16461651. 
11. Lau KKS, Bico J, Teo KBK, Chhowalla M, Amaratunga GAJ, Milne WI, McKinley GH, and Gleason KK. Nano Lett. 2003;3(12):1701-1705.

12. Park SH, Lee SM, Lim HS, Han JT, Lee DR, Shin HS, Jeong Y, Kim J, and Cho JH. ACS Appl. Mater. Inter. 2010;2(3):658-662.

13. Qi H, Wang W, and Li CY. ACS Macro. Lett. 2014;3:675-678.

14. Laird ED and Li CY. Macromolecules 2013;46(8):2877-2891.

15. Li CY, Li L, Cai W, Kodjie SL, and Tenneti KK. Adv. Mater. 2005;17:1198-1202.

16. Li L, Li CY, and Ni CY. J. Am. Chem. Soc. 2006;128(5):1692-1699.

17. Laird ED, Wang W, Cheng S, Li B, Presser V, Dyatkin B, Gogotsi Y, and Li CY. ACS Nano 2012;6(2):1204-1213.

18. Laird ED, Bose RK, Qi H, Lau KKS, and Li CY. ACS Appl. Mater. Inter. 2013; 5(22), 12089-12098.

19. Laird ED, Bose RK, Wang W, Lau KKS, and Li CY. Macromolecular Rapid Communications 2013;34(3):251-256.

20. Nam JH, Lee K-J, Hwang G-S, Kim C-J, and Kaviany M. International J. Heat Mass Trans. 2009;52(11-12):2779-2791.

21. Yu HM, Ziegler C, Oszcipok M, Zobel M, and Hebling C. Electrochimica Acta 2006;51(7):1199-1207.

22. Zhang F-Y, Spernjak D, Prasad AK, and Advani SG. J. Electrochem. Soc. 2007;154(11):B1152-B1157.

23. Jung YC and Bhushan B. J. Micros. 2008;229(1):127-140.

24. Cheng YT, Rodak DE, Angelopoulos A, and Gacek T. Appl. Phys. Lett. 2005;87(19).

25. Zheng Y, Han D, Zhai J, and Jiang L. Appl. Phys. Lett. 2008;92(8). 
26. Varanasi KK, Hsu M, Bhate N, Yang W, and Deng T. Appl. Phys. Lett. 2009;95(9).

27. Li L, Wang W, Laird ED, Li CY, Defaux M, and Ivanov DA. Polymer 2011;52(16):3633-3638.

28. Li L, Li B, Hood MA, and Li CY. Polymer 2009;50(4):953-965.

29. Li CY. J. Poly. Sci. Poly. Phys. 2009;47:2436-2440.

30. Li B, Li LY, Wang BB, and Li CY. Nature Nanotech. 2009;4(6):358-362.

31. Li LY, Li CY, Ni CY, Rong LX, and Hsiao B. Polymer 2007;48(12):3452-3460.

32. Li LY, Li B, Yang GL, and Li CY. Langmuir 2007;23(16):8522-8525.

33. Li L, Yang Y, Yang G, Chen X, Hsiao BS, Chu B, Spanier JE, and Li CY. Nano Lett. 2006;6 1007-1012.

34. Wang W, Laird ED, Gogotsi Y, and Li CY. Carbon 2012;50(5):1769-1775.

35. Wang W, Huang Z, Laird ED, Wang S, and Li CY. Polymer 2015;59:1-9.

36. Wang W and Li CY. ACS Macro Letters 2014;3(2):175-179.

37. As an aside, the authors note that $a$ can be measured directly, but the calculated form was preferred for this work: the electron avalanche in the ESEM under the conditions necessary for this experiment makes direct measurement of $a$ unreliable.

38. Jijun Z, Alper B, Jie H, and Jian Ping L. Nanotechnology 2002;13(2):195.

39. Britz DA and Khlobystov AN. Chemical Society Reviews 2006;35(7):637-659.

40. Controlled microcondensation experiments on molecularly smooth surfaces also show cCAs similar to, but still lower than sCAs. See, e.g., Ref. [23]. Thus we suggest that adsorbed water molecules form a weak buffer layer that lowers the effective solid/liquid interfacial surface tension. 
41. The behaviors of observed droplet shapes defy attempts to reconcile them with first-order calculations based on established principles (see Ref. 17). It was found that calculated values of droplet cCA were consistently higher than observed values. Moreover, the observed droplets should have been higher CA than would be ordinarily observed due to the saturated vapor environment (Ref. 42) and the elevated surface tension due to the high Laplace pressure. Process of elimination suggests that the observed cCA is thus dominated by coalescence of pinned microdroplets.

42. Kayser WV. J. Coll. Inter. Sci. 1976;56(3):622-627.

43. Schonhorn H and Ryan FW. J. Phys. Chem. 1966;70(12):3811-3815. 


\title{
Graphical Abstract
}

\section{Water Nanoondensation on Polymer Single Crystal-Decorated Buckypaper}

\author{
Eric. D. Laird, ${ }^{\dagger} \mathrm{Hao}$ Qi, and Christopher Y. Li ${ }^{*}$
}

Department of Materials Science and Engineering, Drexel University,

Philadelphia, Pennsylvania, 19104, U.S.A.

${ }^{\dagger}$ Present Address: RDRL-WMM-G, 4600 Deer Creek Loop, Aberdeen Proving

Ground, Maryland 21005-5069, U.S.A.

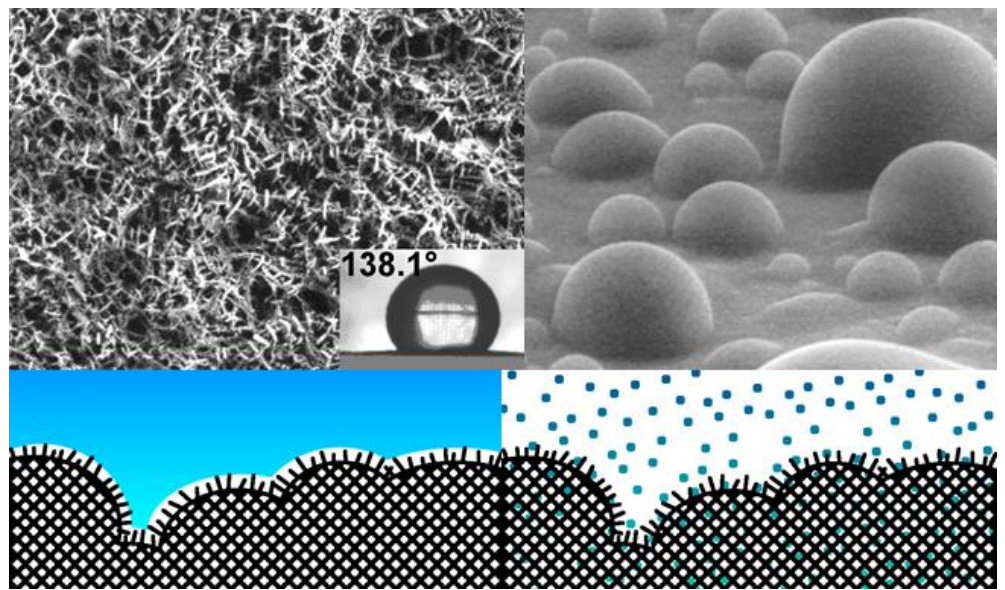

